\title{
Single-stage bilateral pulmonary resections by video-assisted thoracic surgery for multiple small nodules
}

\author{
Feng Yao*, Haitang Yang*, Heng Zhao \\ Department of Thoracic Surgery, Shanghai Chest Hospital, Shanghai Jiaotong University, Shanghai 200030, China \\ Contributions: (I) Conception and design: H Zhao; (II) Administrative support: None; (III) Provision of study materials and patients: None; (IV) \\ Collection and assembly of data: F Yao, H Yang; (V) Data analysis and interpretation: F Yao, H Yang; (VI) Manuscript writing: All authors; (VII) \\ Final approval of manuscript: All authors. \\ *These authors contributed equally to this work. \\ Correspondence to: Heng Zhao. Department of Thoracic Surgery, Shanghai Chest Hospital, Shanghai Jiaotong University, No.241 West Huaihai \\ Road, Shanghai 200030, China. Email: h_zhao28@163.com.
}

\begin{abstract}
Background: Surgical treatment is thought to be the most effective strategy for multiple small nodules. However, in general, one-stage bilateral resection is not recommended due to its highly invasive nature.

Methods: Clinical records of patients undergoing one-stage bilateral resections of multiple pulmonary nodules between January 2009 and September 2014 in a single institution were retrospectively reviewed.

Results: Simultaneous bilateral pulmonary resection by conventional video-assisted thoracic surgery (VATS) was undertaken in 29 patients. Ground glass opacity (GGO) accounted for $71.9 \%$ (46/64) of total lesions, including 26 pure GGO and 20 mixed GGO lesions. One case underwent bilateral lobectomy that was complicated by postoperative dyspnea. Lobar-sublobar (L/SL) resection and bilateral sublobar resection (SL-SL) were conducted in 16 and 12 cases, respectively, and most of these cases had uneventful postoperative courses. There was no significant difference with regard to postoperative complications $(\mathrm{P}=0.703)$, duration of use of chest drains $(\mathrm{P}=0.485)$, between one- and two-stage groups. Mean postoperative follow-up in cases of primary lung cancer was 31.4 (range, 10-51) months. There was neither recurrence nor deaths at final follow-up.

Conclusions: Single-stage bilateral surgery in selected cases with synchronous bilateral multiple nodules (SBMNs) is feasible and associated with satisfactory outcomes.
\end{abstract}

Keywords: Multiple nodules; bilateral; single-stage; video-assisted thoracic surgery (VATS)

Submitted Jul 25, 2015. Accepted for publication Dec 13, 2015.

doi: $10.21037 /$ jtd.2016.02.66

View this article at: http://dx.doi.org/10.21037/jtd.2016.02.66

\section{Introduction}

Owing to advances in diagnostic radiographic methods and increasing awareness of early screening of lung cancers, a higher rate of detection of multiple small pulmonary nodules has been demonstrated, However, difficulties in the preoperative diagnosis (benign or malignant; multiple primary tumors or metastatic tumors) and the choice of therapeutic strategies (surgery, chemotherapy or ongoing follow-up) remain.

Resection allows a sufficient number of specimens for diagnostic differentiation and more favorable long-term survival benefits if the suspicions of malignancy are high. An increasing number of studies has shown benefits from surgical intervention with regard to an early accurate diagnosis and timely treatment with curative intent in patients with synchronous multiple primary lung cancers (SMPLCs) (1,2).

For multiple small pulmonary nodules carrying a high suspicion of malignancy (especially bilateral ones), singlestage surgical treatment with curative intent is considered optimal but appears to be associated with a high risk. In the present study, the feasibility of one-stage surgical treatment 
for synchronous bilateral multiple nodules (SBMNs) is presented and discussed.

\section{Materials and methods}

\section{Patients}

The study was approved by the ethics committee of Shanghai Chest Hospital. During the period of Jan 12009 to Sep 30 2014, cases performed with various pulmonary resections at our department were retrospectively reviewed. Twenty nine cases underwent single-stage bilateral operations of multiple pulmonary nodules were identified. For comparison, 89 patients with bilateral multiple nodules who underwent two-stage operations at the same period were also reviewed. Clinical and histologic features and surgical outcomes were studied.

\section{Surgical procedure}

General anesthesia with double-lumen endotracheal intubation was administered.

Single-stage bilateral surgical treatment by video-assisted thoracic surgery (VATS) was applied based on the following rules: patients with good performance status, patient's informed consent, characteristics of multiple pulmonary foci and individual surgeon's experience. The choice of surgical types comprehensively depends on the site of tumors, the evaluation of cardiopulmonary function reserve and outcomes of intraoperative frozen sections. Single-stage resection was performed with starting on the side of the less invasive resection, such as segmentectomy and wedge resection, generally depending on the size and morphology on preoperative chest computed tomography (CT). For planed bilateral sublobar resections, the priority was given to the right side.

The patient was placed in the lateral decubitus position. After a one-sided procedure, the patient was rotated to the opposite side for a second resection. Cushions were used to lift the patient to avoid kicking or compression to the contralateral chest tube during positioning. Multiple primary lung cancer was diagnosed based on Martini-Melamed criteria (3).

The comprehensive TNM stage was dependent upon the most advanced stage of all lesions in each case of primary lung cancer. All postoperative histopathologicproven adenocarcinomas were reclassified based on the new classification proposed by the International Association for the Study of Lung Cancer, American Thoracic Society, and European Respiratory Society (IASLC/ATS/ERS) (4).

\section{Follow-up}

All patients with pathologically-proven cancers were asked to attend for regular follow-up examinations after surgery, including chest CT scan, abdominal ultrasound examination and serum tumor marker (TM), generally every 6 months.

\section{Statistical analysis}

Continuous variables were presented as mean values \pm standard deviation, and categorical variables were presented as numbers and percentages. Continuous variables were compared between groups using two-sided Student's $t$-tests for normally-distributed data, and two-sided Wilcoxon's rank-sum tests for other data. Categorical variables were compared using contingency table analysis and $\chi^{2}$ tests. For patients with primary non-small lung cancer (NSCLC), survival time was calculated from the date of one-stage surgery and from the second date of two-stage ones. Cumulative survival rates were calculated by the KaplanMeier method.

\section{Results}

\section{Clinical characteristics}

A total of 29 patients [14 men and 15 women; mean age, 56.9 (range, 42-71) years] who underwent singlestage bilateral procedures were identified (Table 1). Eight male patients had a smoking history. Six patients had comorbidities: diabetes mellitus $(\mathrm{n}=3)$, high blood pressure $(n=2)$ and both $(n=2)$. Preoperative forced expiratory volume in 1 second $\left(\mathrm{FEV}_{1}\right)$ and $\mathrm{FEV}_{1} \% /$ mean $\mathrm{FEV}_{1} \%\left(\mathrm{FEV}_{1} \%\right.$ Pred) was $2,596.6 \mathrm{~mL}$ and $103.0 \%$, respectively. During the same period, a bilateral two-stage resection was conducted in 89 cases with multiple lung cancers. There was no significant difference with respect to $\mathrm{FEV}_{1}(\mathrm{P}=0.860)$ and $\mathrm{FEV}_{1} \%$ Pred between one- and two-stage group $(\mathrm{P}=0.189)$ (Table 2). Radiologic review revealed that $62.1 \%(18 / 29)$ of cases had nodules at the bilateral upper lobe (U-U). Ground glass opacity (GGO) accounted for 71.9\% (46/64) of total lesions, including pure GGO (pGGO, n=26) and mixed GGO (mGGO, n=20) lesions. All cases had good performance status, ranging from 0 to 1 .

\section{Surgery and pathology}

Surgical and pathologic characteristics are shown in Table 3. For patients undergoing a one-stage procedure, 
Table 1 Baseline clinical characteristics of 29 cases with synchronous bilateral multiple nodules

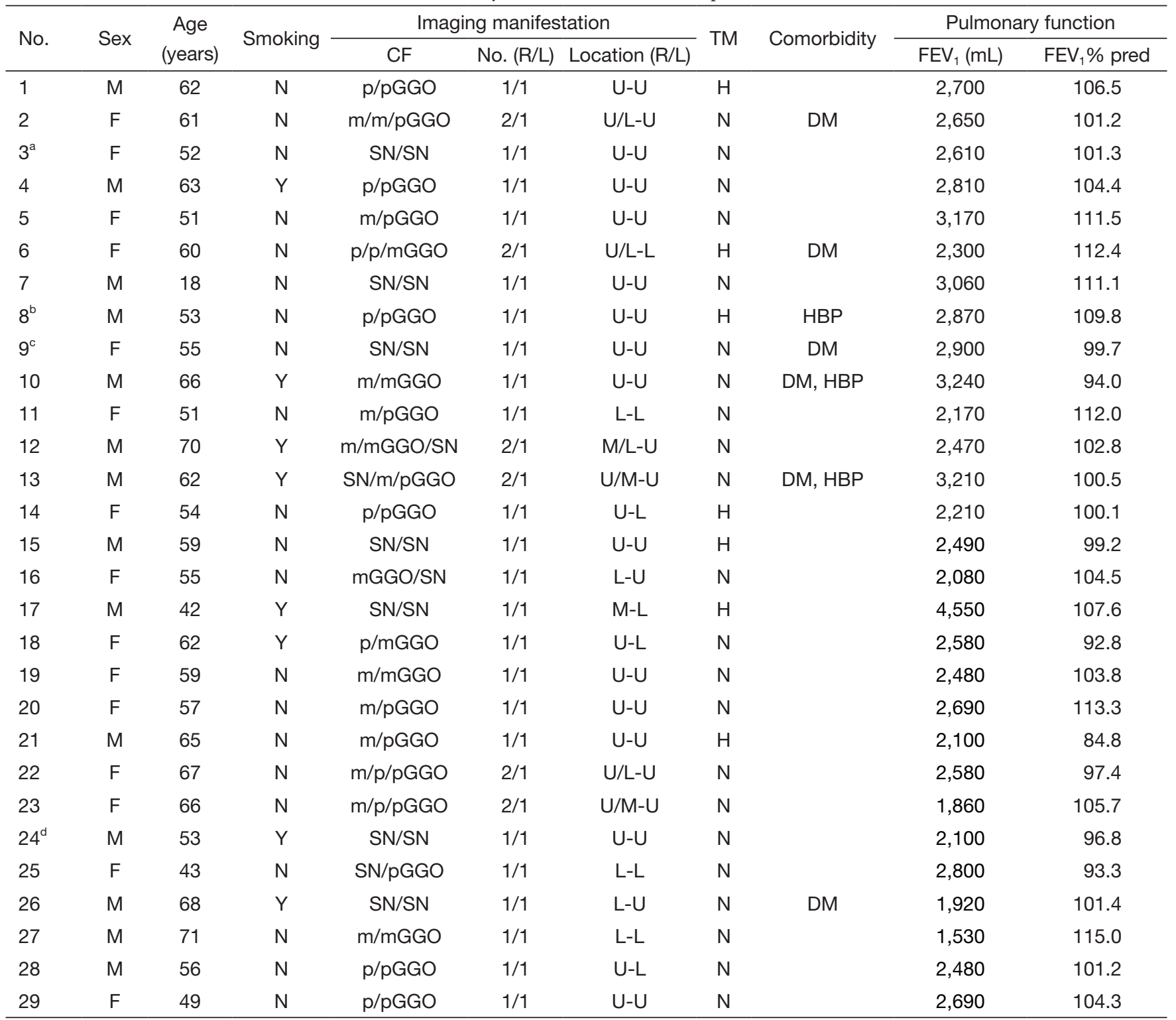

a , breast cancer; ${ }^{b}$, thyroid carcinoma; ${ }^{c}$, parotid carcinoma; ${ }^{d}$, rectal cancer; F, female; M, male; Y, yes; N, no; CF, classification; pGGO, pure ground glass opacity; mGGO, mixed ground glass opacity; SN, solid nodule; G/S, pGGO plus SN; R, right; L, left; U/M/ L, upper/middle/lower lobe; TM, serum tumor marker; $\mathrm{H}$, high; $\mathrm{N}$, normal; FEV ${ }_{1}$, forced expiratory volume in 1 second; FEV $\%$ pred, $\mathrm{FEV}_{1} \% /\left(\right.$ mean $\left.\mathrm{FEV}_{1} \%\right) ; \mathrm{DM}$, diabetes mellitus; HBP, high blood pressure.

bilateral lobectomy (L-L) was done in 1 case, lobectomy and contralateral sublobar resection (L-SL) were done in 16 patients, and bilateral sublobar resection (SL-SL) were conducted in the remaining 12 cases. Mean operation time for patients undergoing L-L/SL and SL-SL was 140.5 and $95.2 \mathrm{~min}$, respectively.

Postoperative histopathology revealed that 22 cases had bilateral primary lung cancer (BPLC) and 3 had bilateral secondary lung cancer. Bilateral benign lesions and primary lung cancer plus a contralateral benign lesion were confirmed in 2 cases each. The most advanced pathologic stage of the primary lung cancer lesion in each patient was classified as IA $(\mathrm{n}=21)$, IB $(\mathrm{n}=1)$, IIA $(\mathrm{n}=1)$, and IIIA $(n=1)$. Retrospective analysis based on the new 
Table 2 Comparisons of clinical data between patients performed with one- and two-stage resections

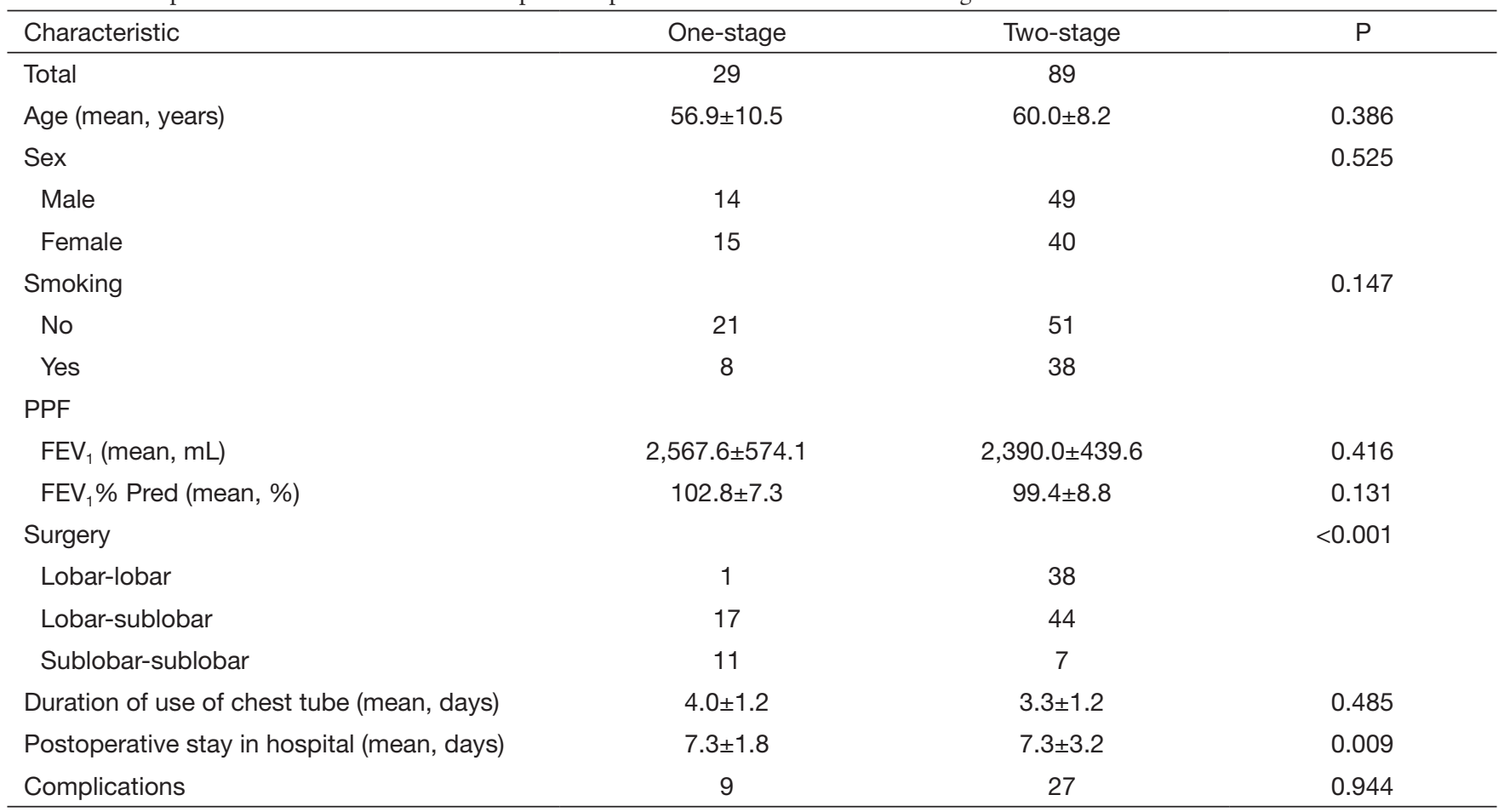

classification proposed by IASLC/ATS/ERS showed that lobectomy with systematic dissection of lymph nodes was conducted for lesions of invasive adenocarcinoma $(n=15)$, minimally invasive adenocarcinoma (MIA, $\mathrm{n}=2)$, and adenocarcinoma in situ (AIS, $\mathrm{n}=1$ ). Considering the size and location of nodules as well as the ipsilateral number of nodules, segmentectomy was selected for lesions of invasive adenocarcinoma $(n=5), \operatorname{MIA}(n=2)$, and AIS $(n=3)$, and wedge resection was considered for lesions of invasive adenocarcinoma $(n=11), \operatorname{MIA}(n=12)$, and AIS $(n=1)$. Visceral pleural invasion (VPI) and/or lymphovascular invasion (LVI) occurred in cases of poorly differentiated adenocarcinoma. That is, solid with mucin-predominant adenocarcinoma (SPA, $\mathrm{n}=2$ ) and acinar-predominant adenocarcinomas (APA, $\mathrm{n}=1$ ).

\section{Postoperative course}

There were no perioperative deaths. Postoperative course was uneventful in $20(69.0 \%)$ cases. Postoperative pneumonia without the requirement of artificial ventilation occurred in $2(6.9 \%)$ patients. Two patients had transient atrial fibrillation. Persistent air leak for $>3$-day duration were observed in $4(13.8 \%)$ patients. Respiratory insufficiency occurred in 1 case that had simultaneous bilateral lobectomy that necessitated 4 days of artificial ventilation. Mean duration of use of a chest drain was $3.5 \pm 1.2$ (range, 2-6) days. The one-stage strategy showed comparable performance with its two-stage counterpart in terms of postoperative complications $(\mathrm{P}=0.944)$ and duration of use of a chest drain $(\mathrm{P}=0.485)$ (Table 2). Overall mean duration of hospitalization after surgery was $7.3 \pm 3.0$ (range, 4-13) days. This value was broken down into 8.0 \pm 1.9 (range, 5-13) days for the L-L/SL group and 6.7 \pm 2.1 (range, 4-9) days for the SL-SL group, respectively, and the difference was significant $(\mathrm{P}=0.004)$. There was a significant decrease in postoperative in-hospital stay for patients who underwent one-stage bilateral resection when compared with the second postoperative hospitalization period of the two-stage group (mean, $7.3 \pm 1.8$ vs. $7.3 \pm 3.2$ days, $\mathrm{P}=0.009)$, owing to a greater prevalence of SL-SL in the one-stage group $(\mathrm{P}<0.001)$. Further analyses based on patients who underwent SL-SL and L-L/SL separately, however, did not reveal a significant difference with respect to postoperative in-hospital stay between the two groups $(\mathrm{P}=0.691$ and $\mathrm{P}=0.065$, respectively). Specifically, in cases who had sequential two-stage bilateral procedures, the second surgery did not significantly prolong postoperative 
Table 3 Surgery and histopathological characteristics $(n=29)$

\begin{tabular}{|c|c|c|c|c|c|c|c|c|c|}
\hline \multirow{2}{*}{ No. } & \multicolumn{5}{|c|}{ Surgical procedure } & \multirow{2}{*}{ Pathology (R/L) } & \multirow{2}{*}{ VPI (R/L) } & \multirow{2}{*}{ LVI (R/L) } & \multirow{2}{*}{$\mathrm{p}$-TNM } \\
\hline & Resection (R/L) & Approach (R/L) & Previous side & LN & OT [total, R/L] & & & & \\
\hline 1 & L-S & $\mathrm{T}-\mathrm{T}$ & $L$ & No & $159[82,77]$ & AIS-MIA & $\mathrm{N}$ & $\mathrm{N}$ & IA \\
\hline 2 & $S / L-W$ & $\mathrm{~T}-\mathrm{T}$ & L & N2 & $160[112,48]$ & APA/APA-MIA & Y & Y & IIIA \\
\hline 3 & W-W & D-D & $\mathrm{R}$ & & $58[32,26]$ & $B C-B C$ & $\mathrm{~N}$ & N & \\
\hline 4 & W-S & D-T & $\mathrm{R}$ & No & $116[31,85]$ & MIA-AIS & $\mathrm{N}$ & $\mathrm{N}$ & IA \\
\hline 5 & L-S & D-T & L & NO & $97[60,37]$ & PPA-PPA & $\mathrm{N}$ & $\mathrm{N}$ & IA \\
\hline 6 & W/S-L & $\mathrm{T}-\mathrm{T}$ & $\mathrm{R}$ & No & $138[81,57]$ & MIA/PPA-PPA & N & $\mathrm{N}$ & IA \\
\hline 7 & L-S & $\mathrm{T}-\mathrm{T}$ & L & No & $215[75,140]$ & B-B & & & \\
\hline 8 & S-S & $\mathrm{T}-\mathrm{T}$ & $\mathrm{R}$ & & $182[105,77]$ & AIS-AIS & $\mathrm{N}$ & $\mathrm{N}$ & \\
\hline 9 & W-W & D-D & $\mathrm{R}$ & & $74[39,35]$ & PC-PC & & & \\
\hline 10 & L-W & T-D & L & No & $75[57,18]$ & SPA-SPA & Y & $\mathrm{N}$ & IB \\
\hline 11 & L-W & $T-D$ & L & No & $132[91,41]$ & SPA-PPA & N & $\mathrm{N}$ & $\mathrm{IA}$ \\
\hline 12 & W-W/W & D-D & $\mathrm{R}$ & No & $101[79,32]$ & APA/APA-LPA & $\mathrm{N}$ & $\mathrm{N}$ & $\mathrm{IA}$ \\
\hline 13 & W-W/W & D-D & $\mathrm{R}$ & No & $118[47,71]$ & LPA/LPA-MIA & $\mathrm{N}$ & N & IA \\
\hline 14 & W-W & D-D & $\mathrm{R}$ & & $40[29,11]$ & B-B & & & \\
\hline 15 & L-S & $\mathrm{T}-\mathrm{T}$ & L & No & $182[90,92]$ & APA-APA & $\mathrm{N}$ & $\mathrm{N}$ & $\mathrm{IA}$ \\
\hline 16 & S-W & T-D & $\mathrm{R}$ & No & $130[90,40]$ & LPA-B & $\mathrm{N}$ & $\mathrm{N}$ & $\mathrm{IA}$ \\
\hline 17 & W-W & D-D & $\mathrm{R}$ & No & $67[38,29]$ & MIA-AIS & $\mathrm{N}$ & $\mathrm{N}$ & IA \\
\hline 18 & W-L & D-T & $\mathrm{R}$ & No & $121[37,84]$ & LPA-LPA & $\mathrm{N}$ & $\mathrm{N}$ & $\mathrm{IA}$ \\
\hline 19 & L-L & $\mathrm{T}-\mathrm{T}$ & $\mathrm{R}$ & NO & $196[86,110]$ & MIA-APA & $\mathrm{N}$ & $\mathrm{N}$ & IA \\
\hline 20 & L-W & $T-D$ & L & No & $145[78,67]$ & LPA-MIA & $\mathrm{N}$ & $\mathrm{N}$ & $\mathrm{IA}$ \\
\hline 21 & L-W & T-D & L & $\mathrm{N} 1$ & $91[62,29]$ & SPA-PPA & $\mathrm{N}$ & $\mathrm{N}$ & IIA \\
\hline 22 & L/W-W & $T-D$ & L & No & $132[87,45]$ & LPA/MIA-MIA & N & $\mathrm{N}$ & $\mathrm{IA}$ \\
\hline 23 & L/W-W & L-D & L & No & $102[69,32]$ & APA/MIA-APA & $\mathrm{N}$ & $\mathrm{N}$ & $\mathrm{IA}$ \\
\hline 24 & W-W & D-D & $\mathrm{R}$ & & $58[31,27]$ & $\mathrm{RC}-\mathrm{RC}$ & & & \\
\hline 25 & S-L & $\mathrm{T}-\mathrm{T}$ & $\mathrm{R}$ & No & $159[68,91]$ & B-LPA & $\mathrm{N}$ & $\mathrm{N}$ & IA \\
\hline 26 & L-W & T-D & L & No & $98[75,23]$ & SPA-SPA & Y & $\mathrm{N}$ & IB \\
\hline 27 & L-S & $\mathrm{T}-\mathrm{T}$ & L & NO & $149[80,69]$ & APA-MIA & $\mathrm{N}$ & $\mathrm{N}$ & IA \\
\hline 28 & W-W & D-D & $\mathrm{R}$ & NO & $101[34,67]$ & MIA-MIA & $\mathrm{N}$ & $\mathrm{N}$ & IA \\
\hline 29 & L-W & $\mathrm{T}-\mathrm{D}$ & L & No & $134[89,45]$ & MIA-MIA & $\mathrm{N}$ & $\mathrm{N}$ & IA \\
\hline
\end{tabular}

L-L, lobectomy-lobectomy; L-S, lobectomy-segmentectomy; L-W, lobectomy-wedge resection; T, three port; D, double port; $\mathrm{U}$, uniport; LN, highest nodal stage; AIS, adenocarcinoma in situ; MIA, minimally invasive adenocarcinoma; LPA, lepidicpredominant adenocarcinoma; PPA, papillary-predominant adenocarcinoma; APA, acinar-predominant adenocarcinoma; SPA, solid with mucin-predominant adenocarcinoma; BC, breast cancer; TC, thyroid carcinoma; B, Benign; PC, parotid carcinoma; RC, rectal cancer; VPI, visceral pleural invasion; LVI, lymphovascular invasion; $p$-TNM, most advanced pathologic TNM stage.

hospitalization compared with the first procedure in the same patient (mean, $7.4 \pm 1.7$ vs. $8.0 \pm 2.4$ days, $\mathrm{P}=0.236$ ). This phenomenon was attributable mainly to more limited resections in the second procedure.

\section{Follow-up and survival}

Mean duration of follow-up in cases with primary lung cancer was 31.4 (range, 10-51) months. There was neither recurrence nor death at final follow-up. 


\section{Discussion}

Studies have shown that patients with bilateral earlystage NSCLC can benefit more from bilateral resection with curative intent than those receiving a combination of surgery and chemotherapy or radiotherapy (5). Theoretically, simultaneous bilateral resection with curative intent is ideal compared with a two-stage procedure in terms of decreasing the risk of disease progression. However, given that simultaneous bilateral procedures are highly invasive with potentially severe complications this choice is not considered feasible by most thoracic surgeons even if they are experienced in VATS (a very minimally invasive procedure).

Published series on the feasibility and safety of singlestage bilateral procedures via multi-portal VATS or median sternotomy in bilateral SMPLC cases are rare $(6,7)$. In our series, traditional multi-portal VATS (which is a wellestablished approach for undertaking various types of radical resections for lung cancer) was used for singlestage bilateral procedures. There were no deaths, and the postoperative course was uneventful in $69.0 \%$ of cases. Of those with postoperative complications, all had a rapid recovery except 1 case that had a bilateral lobectomy. In addition, a significant difference was not observed with respect to the various types of resections between one- and two-stage groups (L-L/SL, $\mathrm{P}=0.065$; SL-SL, $\mathrm{P}=0.691$ ). Furthermore, the one-stage strategy showed comparable performance with its two-stage counterpart in terms of postoperative complications $(\mathrm{P}=0.944)$ and duration of use of chest drains $(\mathrm{P}=0.485)$, but the latter would increase the economic burden.

In the past decade, an increasing number of reports focusing on the applications of uniportal VATS in thoracic procedures has been published (8-11). Despite its seemingly less minimally invasive nature, whether there are differences in outcomes of uniportal VATS compared with multiportal VATS is controversial (12-14). Recently, Liu and colleagues reported successful single-stage bilateral pulmonary resections (two left-sided wedge resections plus a right upper lobectomy in one patient, and a left lower lobe superior segmentectomy plus a right upper lobectomy in another patient) by uniportal VATS in 2 cases with SMPLC (15). Uniportal VATS appeared to have much longer operation time (260 and $285 \mathrm{~min}$, respectively, in the report by Liu and colleagues) than a traditional multiportal procedure (mean, 140.5 and $95.2 \mathrm{~min}$ in our cases who had L-L/SL and SL/SL, respectively) with respect to lobectomy or segmentectomy. In addition, the highly technical requirements and potentially problematic intraoperative complications associated with uniportal VATS compared with the multiportal counterpart make widespread use very difficult. Uniportal VATS might be, to some extent, helpful for the relief of postoperative pain but the adverse effects of a longer procedure and anesthesia time in patients undergoing lobectomy or segmentectomy might offset the benefits of less pain associated with uniportal VATS. For patients with a planned wedge resection, combination of uniportal VATS and preoperative location methods for small pulmonary nodules (e.g., hook wire) might be preferable (6) whereas, for lobectomy or segmentectomy, a multiportal approach might be more likely. Further studies based on larger samples are required to weigh the benefits of multiportal $v s$. uniportal VATS for patients undergoing single-stage bilateral pulmonary resections.

An optimal surgical method for SBMPLC is lacking (16-18). Lobectomy remains first-line treatment for earlystage NSCLC but a single-stage bilateral lobectomy is not recommended due to the severe trauma imparted. The only case who had a one-stage bilateral lobectomy in our series suffered complications necessitating artificial ventilation for 4 days. Sublobar resection has been shown to be equivalent to lobectomy for clinical stage-IA solitary lung cancers (19). However, Zuin and colleagues (18) showed that patients who had a lobectomy showed better 5 -year survival than those who underwent sublobar resection $(57.5 \%$ and $36 \%$, respectively, $\mathrm{P}=0.016$ ). Unfortunately, the classification of lesions employed was not known given the different prognoses of various subtypes of lesions (4). For patients with small-sized and well-differentiated lesions, such as pGGO $<2 \mathrm{~cm}$ and AIS/MIA, 5-year survival might not necessarily be compromised by sublobar resections (segmentectomy and wedge resection) $(19,20)$. Thus, a one-stage bilateral procedure might be suitable. For patients with poorly differentiated (e.g., SPA and mucinous adenocarcinoma) or bilateral advanced-stage lesions (e.g., mixed GGO containing $>50 \%$ solid component), GGO located deep or multiple nodules in one lobe, a two-stage bilateral lobectomy with a short of interval 4-6 weeks might be preferred.

\section{Conclusions}

Satisfactory outcomes can be achieved by single-stage bilateral surgery in selected cases with SBMNs. Multiportal VATS may be preferred for lesions for which lobectomy or segmentectomy is planned. 


\section{Acknowledgements}

Funding: The work has been supported by the grants from National Natural Science Foundation of China (81572693), and Science and Technology Commission of Shanghai Municipality (10411967800).

\section{Footnote}

Conflicts of Interest: The authors have no conflicts of interest to declare.

\section{References}

1. Kocaturk CI, Gunluoglu MZ, Cansever L, et al. Survival and prognostic factors in surgically resected synchronous multiple primary lung cancers. Eur J Cardiothorac Surg 2011;39:160-6.

2. Jiang L, He J, Shi X, et al. Prognosis of synchronous and metachronous multiple primary lung cancers: systematic review and meta-analysis. Lung Cancer 2015;87:303-10.

3. Martini N, Melamed MR. Multiple primary lung cancers. J Thorac Cardiovasc Surg 1975;70:606-12.

4. Travis WD, Brambilla E, Noguchi M, et al. International association for the study of lung cancer/american thoracic society/european respiratory society international multidisciplinary classification of lung adenocarcinoma. J Thorac Oncol 2011;6:244-85.

5. Iino K, Oda M, Tsunezuka Y, et al. Treatment for bilateral multiple lung cancers. Kyobu Geka 2002;55:443-8; discussion 448-50.

6. Mun M, Kohno T. Single-stage surgical treatment of synchronous bilateral multiple lung cancers. Ann Thorac Surg 2007;83:1146-51.

7. Hosaka Y, Motono N, Satou S, et al. Single-stage operation for synchronous bilateral multiple lung cancer through median sternotomy. Kyobu Geka 2009;62:456-9.

8. Gonzalez-Rivas D, Paradela M, Fernandez R, et al. Uniportal video-assisted thoracoscopic lobectomy: two years of experience. Ann Thorac Surg 2013;95:426-32.

9. Rocco G, Martucci N, La Manna C, et al. Ten-year experience on 644 patients undergoing single-port (uniportal) video-assisted thoracoscopic surgery. Ann Thorac Surg 2013;96:434-8.

10. Chen CH, Chang H, Tai CY, et al. Bilateral pulmonary metastectomy through a unilateral single-port thoracoscopic approach. J Thorac Dis 2014;6:143-7.

11. Feng $M$, Shen $Y$, Wang $H$, et al. Uniportal video assisted thoracoscopic lobectomy: primary experience from an Eastern center. J Thorac Dis 2014;6:1751-6.

12. Akter F, Routledge T, Toufektzian L, et al. In minor and major thoracic procedures is uniport superior to multiport video-assisted thoracoscopic surgery? Interact Cardiovasc Thorac Surg 2015;20:550-5.

13. Chung JH, Choi YS, Cho JH, et al. Uniportal videoassisted thoracoscopic lobectomy: an alternative to conventional thoracoscopic lobectomy in lung cancer surgery? Interact Cardiovasc Thorac Surg 2015;20:813-9.

14. Jimenez MF. Uniportal versus standard video-assisted thoracoscopic surgery for lung lobectomy: changing the standards requires scientific evidence. Eur J Cardiothorac Surg 2015;47:916.

15. Liu C, Ma L, Lin F, et al. Single-staged uniportal VATS major pulmonary resection for bilateral synchronous multiple primary lung cancers. J Thorac Dis 2014;6:1315-8.

16. Baisi A, De Simone M, Cioffi U, et al. Should pulmonary lobectomy be replaced by sublobar resection in patients with stage I non-small cell lung cancer? J Thorac Cardiovasc Surg 2014;147:1997-8.

17. Toufektzian L, Attia R, Veres L. Does the extent of resection affect survival in patients with synchronous multiple primary lung cancers undergoing curative surgery? Interact Cardiovasc Thorac Surg 2014;19:1059-64.

18. Zuin A, Andriolo LG, Marulli G, et al. Is lobectomy really more effective than sublobar resection in the surgical treatment of second primary lung cancer? Eur J Cardiothorac Surg 2013;44:e120-5; discussion e125.

19. Altorki NK, Yip R, Hanaoka T, et al. Sublobar resection is equivalent to lobectomy for clinical stage 1A lung cancer in solid nodules. J Thorac Cardiovasc Surg 2014;147:75462; Discussion 762-4.

20. Shimada Y, Saji H, Otani K, et al. Survival of a surgical series of lung cancer patients with synchronous multiple ground-glass opacities, and the management of their residual lesions. Lung Cancer 2015;88:174-80.

Cite this article as: Yao F, Yang H, Zhao H. Single-stage bilateral pulmonary resections by video-assisted thoracic surgery for multiple small nodules. J Thorac Dis 2016;8(3):469475. doi: $10.21037 /$ jtd.2016.02.66 